\title{
El estrés académico y la educación en aulas virtuales en estudiantes universitarios
}

Academic stress and education in virtual classrooms in university students

1 Emily Pamela Salazar Padilla

https://orcid.org/0000-0002-9530-4255

Universidad Técnica de Ambato, Carrera de Psicopedagogía, Ambato, Ecuador, esalazar2870@uta.edu.ec

2 Karen Cristina Ojeda

https://orcid.org/0000-0002-7750-8190

Universidad Técnica de Ambato, Facultad de Ciencias Humanas y de la Educación,

Ambato, Ecuador,

kojeda0843@uta.edu.ec

3 Xiomara del Rocío Tabares Rosero (iD) https://orcid.org/0000-0003-2605-3509

Universidad Técnica de Ambato, Facultad de Ciencias Humanas y de la Educación,

Ambato, Ecuador.

xdr.tabares@uta.edu.ec

4 María Belén Morales Jaramillo (iD) https://orcid.org/0000-0003-2466-1885

Universidad Técnica de Ambato, Facultad de Ciencias de la Educación, Carrera de

Psicopedagogía. Ambato, Ecuador.

mb.morales@uta.edu.ec

Artículo de Investigación Científica y Tecnológica

Enviado: 24/12/2021

Revisado: 29/12/2021

Aceptado: 12/01/2022

Publicado:08/03/2023

DOI: https://doi.org/10.33262/concienciadigital.v6i1.4.2013

Cítese. Salazar Padilla, E. P., Cristina Ojeda, K., Tabares Rosero, X. del R., \& Morales Jaramillo, M. B. (2023). El estrés académico y la educación en aulas virtuales en estudiantes universitarios. ConcienciaDigital, 6(1.4), 551-565. https://doi.org/10.33262/concienciadigital.v6i1.4.2013

CONCIENCIA DIGITAL, es una Revista Multidisciplinar, Trimestral, que se publicará en soporte electrónico tiene como misión contribuir a la formación de profesionales competentes con visión humanística y crítica que sean capaces de exponer sus resultados investigativos y científicos en la misma medida que se promueva mediante su intervención cambios positivos en la sociedad. https://concienciadigital.org .

La revista es editada por la Editorial Ciencia Digital (Editorial de prestigio registrada en la Cámara Ecuatoriana de Libro con No de Afiliación 663) www.celibro.org.ec 


Palabras
claves:
estrés,
educación,
capacidad,
compresión,
aulas virtuales,
plataformas,
internet,
recursos
tecnológicos,
factores
estresantes.

Keywords:

stress, education, compression, virtual classrooms, platforms, internet, technological resources, stressors.
Resumen

Introducción. En el transcurso de la vida estudiantil, los universitarios se han visto expuestos a varios cambios, en especial en estos últimos años donde la presión y la falta de adaptación a la educación virtual han desencadenado varias repercusiones dentro de su salud mental, principalmente relacionados al estrés, este afectando su desempeño académico. La educación virtual ha evidenciado debilidades como la falta de tiempo para el desarrollo de trabajo autónomo, la carencia de computadores actualizados e internet y la debilidad en la metodología y didáctica por parte de los docentes para el acceso y uso de ciertos recursos tecnológicos, plataformas, o el desarrollo de trabajos. Estos indicadores junto a la realidad de la pandemia COVID 19, ha incrementado de manera significativa los niveles de estrés en general y académico en particular. Objetivo. Analizar si el contexto académico virtual está generando estrés en los estudiantes universitarios. Metodología. Se aplica un enfoque mixto ya que buscamos analizar, describir la relación del estrés académico con la educación en aulas virtuales, un diseño descriptivo, una modalidad de campo y bibliográfica ya que la información fue recolectada de fuentes primarias. Resultados. Según los hallazgos los estudiantes presentan altos niveles de estrés asociado a las problemáticas que se presentan al usar las aulas virtuales. Conclusión. Existe relación entre el estrés académico y la educación en aulas virtuales, por lo cual es necesario la aplicación de estrategias de intervención que permitan la prevención y disminución del estrés académico en los estudiantes.

\section{Abstract}

Introduction. During student life, university students have been exposed to various changes, especially in recent years where pressure and lack of adaptation to virtual education have triggered various repercussions within their mental health, mainly related to stress, is affecting their academic performance. Virtual education has shown weaknesses such as the lack of time for the development of autonomous work, the lack of updated computers and the internet, and the weakness in the methodology and didactics on the part of teachers for the access and use of certain technological resources, platforms, or the development of jobs. These indicators, together with the reality of the COVID 19 pandemic, have 
significantly increased levels of stress in general and academic. Target. Analyze if the virtual academic context is generating stress in university students. Methodology. A mixed approach is applied since we seek to analyze, describe the relationship of academic stress with education in virtual classrooms, a descriptive design, a field, and bibliographic modality since the information was collected from primary sources. Results. According to the findings, the students present high levels of stress associated with the problems that arise when using virtual classrooms. Conclusion. There is a relationship between academic stress and education in virtual classrooms, which is why it is necessary to apply intervention strategies that allow the prevention and reduction of academic stress in students.

\section{Introducción}

Estrés. - El estrés es la manera en la que el cerebro y el cuerpo responden ante cualquier tipo de desafío. Un estrés elevado se relaciona con la muerte neuronal por envejecimiento, alteración hormonal producida por la elevación del cortisol, cambios emocionales, físicos, etc., todas estas características influyen en la creatividad, motivación y en general la calidad de vida de la persona (Bairero, 2017). La calidad de vida se la asocia con la felicidad y bienestar, por ende, genera satisfacción a una persona si su desarrollo está acorde a su contexto, es decir, cultura, valores y normas impuestas por la sociedad (Perren \& Lamfre, 2017). Es la capacidad de organización de las actividades que permite conservar o incrementar el bienestar físico y mental de la persona, esto tiene el objetivo de lograr un equilibrio en la vida, el cual se logra a través de la práctica de actividad física dos o tres veces por semana de manera regular, este tipo de actividad trae consigo efectos positivos en la salud orgánica, funcional, física y psicológica (Pérez \& Díaz, 2019).

\section{Estrés académico}

El estrés académico es la reacción normal que los estudiantes tienen frente a las diversas exigencias y demandas a las que se enfrentan en la Universidad, tales como pruebas, exámenes, trabajos, presentaciones, etc. Esta reacción les activa y les moviliza para responder con eficacia y conseguir sus metas y objetivos en cuanto se refiere al estrés. Además, se caracteriza por ser un proceso metódico, adaptativo y psicológico, se presenta por la incapacidad e incertidumbre que se genera por diferentes causas, algunas de ellas son la acumulación de labores, gran cantidad de compromisos o malestar en el ambiente laboral, entre otras (Indacochea et al., 2021). Sin embargo, cuando las exigencias del 
medio sobrepasan los recursos internos, se produce el distrés, desencadenando un desequilibrio fisiológico, psicológico, académico y social, perjudicando a los estudiantes ya que evidencian un bajo rendimiento en las distintas áreas de estudio, sumado a esto se puede experimentar dentro del entorno familiar tensiones o falta de apoyo para realizar tareas aumentando su nivel de estrés, lamentablemente estas situaciones de estrés o desmotivación no está siendo percibida por los padres de familia siendo estos el núcleo para la interacción.

El estrés también abarca en primer lugar a la ansiedad como respuesta de nuestro organismo en torno a las tensiones y alteración de nuestro estado mental, causada por los estímulos que afectan el equilibrio emocional y mental, esto genera directamente en los estudiantes adolescentes cambios de humor fuertes, retraimiento y descontento con lo que hacen día a día. Una sobrecarga en tareas, los exámenes sin previo entendimiento, carencia de clima social positivo y dificultades en la participación han creado un temor y exhausto estrés cotidiano (Ticona et al., 2021).

Como estudiantes se ven en la obligación de afrontar diferentes despliegues y esto conlleva a adaptarse a estresores diferentes conforme pasan a niveles más altos En el mundo uno de cada cuatro individuos sufre de problemas de estrés, se estima que en cada ciudad existe un 50\% de personas que tienen alguna enfermedad de salud mental relacionadas al estrés. Según Maldonado, Hidalgo y Otero un nivel elevado de estrés académico altera el sistema de respuestas del individuo a nivel cognitivo, motor y fisiológico. En consecuencia, el estrés se origina por la relación entre el individuo y el entorno, si este es amenazante buscara como confrontarlo (Berrío \& Mazo, 2018).

Hay que mencionar que existen consecuencias ante la presencia del estrés en los estudiantes, como pueden ser incremento de la frecuencia cardiaca, transpiración, tensión muscular de piernas o brazos, respiración lenta, falta de sueño que puede acarrear un trastorno, fatiga crónica, cefalea y problemas de digestión; todas las reacciones afectan de manera conductual al desempeño, pueden aislarse, también puede acarrear al consumo de sustancias como alcohol, drogas, tabaco y otros, aumentado el nerviosismo, la falta de apetito y sueño (Silva et al., 2020).

La vida académica requiere en los estudiantes esfuerzos para adaptarse a demandas y metas que se deben alcanzar durante el año lectivo, enfrentarse a situaciones de incertidumbre, sobrecarga académica y alcanzar o no sus expectativas han puesto a la vista problemas de este, añadido a esto podemos sumarle condiciones psicosociales como problemas financieros, familiares, tiempo limitado para compartir con la familia o amigos de su entorno, con esto se denotara más los niveles de estrés en el sujeto (Emiro, Amador, \& Castañeda, 2020).

Cuando los estudiantes tienden a estar acostumbrados a un tipo de interacción familiar, 
social y cultural tienden a desarrollarse según su contexto, cuando su entorno es difuso y alterado los estudiantes adoptan los comportamiento, pensamientos y emociones según su realidad de interacción, lo cual es contraproducente y dañina para su salud, esto es suficiente para causar malestar y tensión en las personas sobre todo cuando están en la adolescencia. El estrés puede ser percibido como una reorganización de patrones conductuales que pueden llevar al éxito si los sujetos son resilientes, pero cuando el estrés altera la homeostasis psíquica individual, pueden originar pérdida de control sobre las situaciones, resultando abrumadoras (Méndez et al., 2018).

Consecuencias del estrés académico. - Como respuesta psicológica del estrés, la persona presenta cambios de humor drásticos, dificultad para controlar emociones, baja autoestima, etc., que afectan la capacidad para concentrarse en una actividad determinada. En cuanto a lo fisiológico, las personas pueden comenzar a presentar síntomas psicosomáticos, como los cambios corporales, en donde el organismo incrementa el potencial físico para adaptarse de mejor manera a su contexto. La respuesta comportamental del estrés tiene relación con el contexto en el que se desarrolla el individuo y su estilo de vida, ya que el comportamiento puede ayudar a mantener una rutina de vida saludable para evitar y reducir el estrés, como es realizar ejercicio mínimo 3 veces a la semana, mantener una buena alimentación, etc., de lo contrario existe mayor probabilidad de desencadenar estrés crónico y por ende caer en adicciones como el consumo excesivo de alcohol, tabaco, drogas e incluso llegar a aislarse totalmente.

\section{Aulas virtuales}

La educación se ha convertido en un espacio en el cual las tecnologías de la información se han vuelto el motor de arranque para transformar currículos, planes de estudio, técnicas, estrategias, modalidades educativas y toda la información, los nuevos entornos de aprendizaje han generado nuevos hábitos de vida las cuales han causado ciertas necesidades para la población, por causa de diversas índoles que atraviesan los estudiantes día a día dentro de las aulas (Toledo, 2019).

Podemos entender que el aula virtual es un tipo de entorno donde se dan practicas comunicacionales, comparten recursos y se construye en una comunidad bienestar y conocimiento, este espacio social estará conformado por estudiantes, tutores y docentes, demandando tecnología para poder moverse a través de este, requiriendo que en los estudiantes no se produzca un tipo de limitación, estrés o desmotivación por no obtener herramientas para ser partícipe de este y mejorar su ambiente (Svensson, 2018).

Las aulas virtuales en los últimos años se han planteado atender a todas las modalidades, como son la enseñanza primaria, secundaria, bachillerato y también en las universidades, tienen un carácter complementario. Para la actividad docente se gestiona los materiales a utilizar, los apuntes, los documentos de estudio, los programas, las horas y las 
calificaciones que se asignaran en base a los formatos que tengan ya establecidos. Hay que aclarar que los docentes son los que ayudaran y formaran al estudiante de manera que se consideran como el recurso de apoyo más importante (Salvat, 2017).

La educación se ha visto modificada en el aspecto del uso de plataformas donde se realizan y envían las tareas, y también donde se encuentra el contenido didáctico para un manejo total de las actividades. Los docentes y estudiantes han cambiado su manera de trabajo ya que ahora se ve a través de un sistema computacional con conexión a internet. Los docentes facilitan videos, audios, tomando en cuenta la mejor manera para llegar con un aprendizaje significativo, juntamente con los estudiantes aplicaran la pedagogía critica, que les permite crear sus propios análisis a partir de conocimientos ya adquiridos en el aula y en forma autoaprendizaje (Sánchez, 2020).

Se ha necesitado la preparación de los docentes, donde algunos de estos se han visto afectados por las tecnologías que se han vuelto nuevas para ellos, si bien sabemos el mayor responsable es el docente, comprometiéndose para planificar no solo material teórico, sino también la manera para hacer rendir de la mejor manera las herramientas que garanticen un buen desempeño profesional inspirándoles motivación, y asignando un rol totalmente activo al estudiante, convirtiéndolo en un ser responsable y con proyección hacia un ambiente de construcción (Gil, et al., 2019).

Tanto docentes como estudiantes han visto estas herramientas como plataformas y demás como un apoyo de enseñanza-aprendizaje, donde también el estudiante debe generar independencia para desarrollar actividades de manera autónoma, aprovechando la información que se obtiene de las páginas webs de internet, haciendo sus tareas ya sea de manera individual o en equipos donde se pretende compartir información y complementarse entre sí (Navarrete, et al., 2019).

\section{Educación en aulas virtuales}

El internet ha ido evolucionando constantemente a lo largo del tiempo, se ha normalizado la comunicación a través de los dispositivos electrónicos y se han presentado como un apoyo para la educación, en dónde se pone en práctica lo virtual y presencial; esto se ha presenciado más en los últimos años debido a la pandemia por Covid-19, el confinamiento que se vivió durante el primer año cambió el estilo de vida de las personas a nivel mundial; la tecnología pasó a ser un aliado en el ámbito laboral, educativo y social, ya que se convirtió en una herramienta fundamental para facilitar su interacción. En el ámbito educativo ayudó a fomentar el aprendizaje autónomo y colaborativo entre docentes y alumnos, dejando de lado la escuela tradicionalista en dónde el profesor tenía el control y una participación activa, mientras que la participación del estudiante era pasiva y se limitaba a escuchar lo que decía el docente, sin opción a cuestionarlo, por lo tanto el cambio ha sido radical, tanto que se ha tenido que implementar nuevas estrategias 
pedagógicas y romper la brecha digital que ha existido a lo largo de los años, haciendo uso de diferentes plataformas virtuales, recursos educativos gratuitos y otras herramientas que permiten al proceso educativo emplear nuevos roles dejando de lado los tradicionales (Martínez \& Jiménez, 2020).

Tanto los decentes como los estudiante se han visto en la obligatoriedad de aprender nuevas formas de cómo enseñar y como aprender dentro del nuevo contexto, como mencionamos antes esto ha repercutido en modificaciones en los estilos de enseñanzaaprendizaje dentro de todas la áreas y materias que se dan en cada semestre, considerando esta modalidad virtual los docentes y sus competencias ya antes adquiridas han tenido que desarrollarse de otra manera utilizando completamente recursos tecnológicos para así acoplarse a los niveles de enseñanza requerida actualmente (Carrasco, et al., 2021).

Las aulas virtuales tienen como objetivo fortalecer el aprendizaje autónomo, el cual se refiere a la capacidad que tiene el estudiante para intervenir activamente durante la clase, estableciendo momentos significativos que ayudan a su formación, aportando conocimientos y despejando dudas (Solórzano, 2017), otro de sus objetivos es el trabajo colaborativo, en donde se logra resolver problemas de manera conjunta, lo que da como resultado un mayor alcance del que se logra individualmente, por lo tanto la colaboración es la clave para el desarrollo de competencias de las personas, ya que se convierten en una fuente de motivación y creatividad, de esta manera se transmite conocimientos importantes.. La creatividad es otro objetivo de las aulas virtuales, se propone generar ideas originales en un entorno social determinado, en la educación es un factor importante, ya que permite tener motivación e interés al momento de adquirir nueva información, la cual debe ser novedosa y útil, su último objetivo es el pensamiento crítico, que se caracteriza por estar constituido por habilidades cognitivas de alto nivel. Para lograrlo, se requiere que el estudiante domine principalmente los procesos de metacognición que constituyen una de las fortalezas potenciadas cuando se aplican las metodologías propias del logro de aprendizaje significativo, como clave para aprender a aprender y aprender a pensar.

Teniendo en cuenta estos objetivos, las aulas virtuales se basan en la innovación y calidad educativa, las mismas que se caracterizan por ser flexibles, integrales, versátiles, potenciales y diversas, el docente es el encargado de brindar la información, utilizar recursos didácticos, estimular habilidades y competencias en sus estudiantes y fomentar el aprendizaje autónomo y cooperativo entre pares (Martínez \& Jiménez, 2020).

\section{Metodología}

En el siguiente estudio se realizó bajo una investigación descriptiva, de un enfoque mixto, debido a que se consideró fundamental utilizar distintas técnicas de recolección de datos juntamente con la combinación del enfoque cualitativo y cuantitativo de investigación, la integración de ambas ayudará de una manera más adecuada a comprender los conceptos 
y responder del modo más enriquecedor al tema de estudio. La investigación en un nivel correlacionar, con una modalidad bibliográfica y de campo, ya que no solo persigue describir o acercarse al problema, sino también intenta encontrar las causas por las cuales se dan o se sigue produciendo un problema.

Para la recopilación de datos se empleó la investigación de campo, se trabajódirectamente con los estudiantes de séptimo semestre de la Universidad Técnica de Ambato, quienes expresaron el nivel de estrés académico que tienen durante el semestre mediante el instrumento que se aplicó. El primer test aplicado fue dirigido a calificar el estrés académico y el segundo, que fue un cuestionario hace alusión a las aulas virtuales, a los cuales se aplicó la correlación de Spearman. Los resultados que arrojo nos ayudan a verificar si existen percusiones negativas del estrés sobre los estudiantes en sus estudios dentro del contexto virtual.

\section{Resultados}

Para el procesamiento y análisis de la información proporcionada por los estudiantes universitarios, en el presente trabajo de investigación, fue necesario aplicar un test juntamente con un cuestionario, en un total de 44 ítems, seleccionando únicamente los más notables.

\section{Estrés académico}

\section{Figura 1}

Preguntas relacionadas con el test de estrés académico
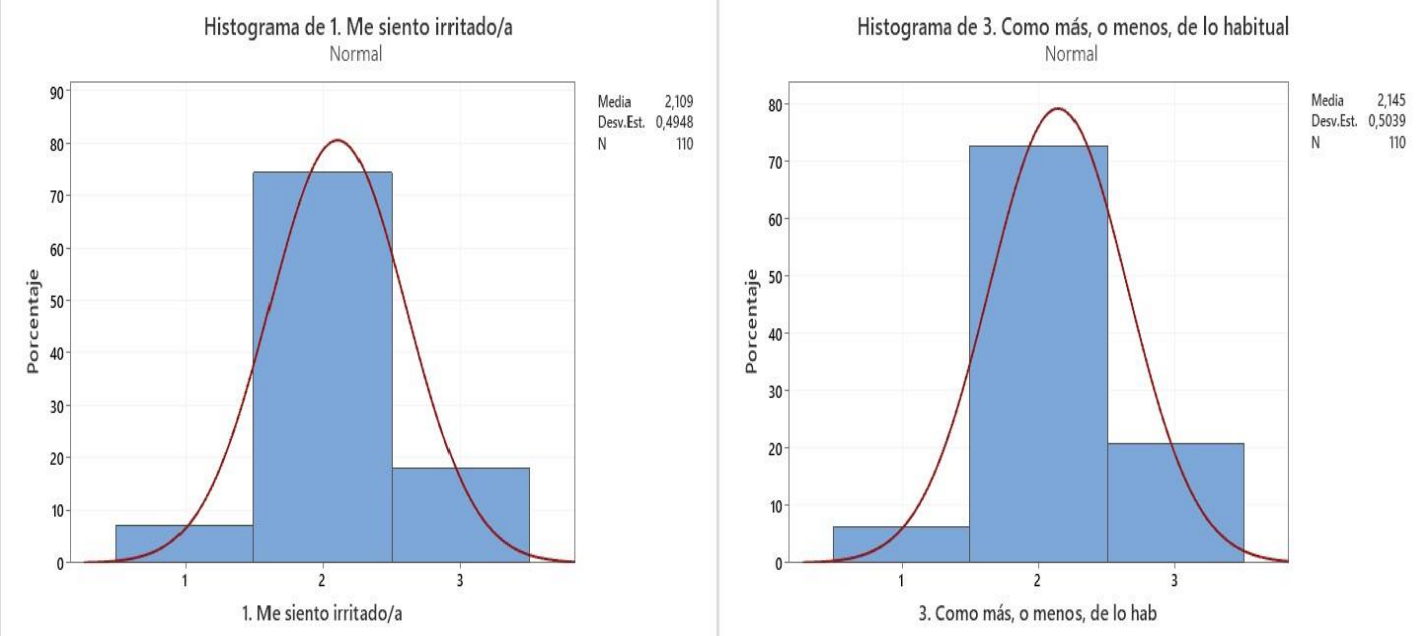

Nota: Alto nivel de estrés académico en estudiantes universitarios.

Fuente: Estudio de estrés académico

Los estudiantes encuestados provienen de la carrera de psicopedagogía, de la Universidad Técnica de Ambato, y se evaluaron con el cuestionario de estrés adolescente, el $80 \%$ 
corresponde a 88 estudiantes que presentan un alto nivel de estrés, el 19\% corresponde a 21 estudiantes, presentan un bajo nivel de estrés; mientras que el resto de los encuestados no presentan estrés, en un conjunto son 110 personas que han formado parte del estudio sobre el estrés académico.

\section{Tabla 1}

\section{Estudiantes encuestados por estrés académico}

\begin{tabular}{|c|c|c|c|c|c|c|c|}
\hline \multicolumn{3}{|c|}{ Descripción } & \multirow{2}{*}{$\frac{\text { Siempre \% }}{18 \%}$} & \multicolumn{2}{|c|}{ A veces $\%$} & \multicolumn{2}{|c|}{ Nunca $\%$} \\
\hline 1 & Me siento irritado/a & 20 & & 82 & $75 \%$ & 8 & $7 \%$ \\
\hline 2 & Como más, o menos, de lo habitual & 23 & $21 \%$ & 80 & $73 \%$ & 7 & $6 \%$ \\
\hline 3 & Dejo las cosas para otro día & 12 & $11 \%$ & 86 & $78 \%$ & 12 & $11 \%$ \\
\hline 4 & Me siento asustado/a & 18 & $16 \%$ & 78 & $71 \%$ & 14 & $13 \%$ \\
\hline 5 & Me siento preocupado/a & 39 & $35 \%$ & 68 & $62 \%$ & 3 & $3 \%$ \\
\hline 6 & Me siento ansioso/a & 29 & $26 \%$ & 65 & $59 \%$ & 16 & $15 \%$ \\
\hline 7 & Actúo a la defensiva con los demás & 13 & $12 \%$ & 56 & $51 \%$ & 41 & $37 \%$ \\
\hline 8 & Me siento agobiado/a & 16 & $15 \%$ & 76 & $69 \%$ & 18 & $16 \%$ \\
\hline 9 & Siento sudor frío & 3 & $3 \%$ & 53 & $48 \%$ & 54 & $49 \%$ \\
\hline 10 & Tengo picores por todo el cuerpo & 6 & $5 \%$ & 39 & $35 \%$ & 65 & $59 \%$ \\
\hline 11 & Me siento incapaz de afrontar el institut & & $6 \%$ & 65 & $59 \%$ & 38 & $35 \%$ \\
\hline 12 & Me siento indeciso/a & 16 & $15 \%$ & 83 & $75 \%$ & 11 & $10 \%$ \\
\hline 13 & Descuido mis amistades & 15 & $14 \%$ & 74 & $67 \%$ & 21 & $19 \%$ \\
\hline 14 & Pierdo la voz o me quedo afónico/a & 4 & $4 \%$ & 36 & $33 \%$ & 70 & $64 \%$ \\
\hline 15 & Siento que no sé qué hacer & 19 & $17 \%$ & 70 & $64 \%$ & 21 & $19 \%$ \\
\hline 16 & Hablo mal de mis compañeros/as... & 5 & $5 \%$ & 27 & $25 \%$ & 78 & $71 \%$ \\
\hline 17 & Tengo palpitaciones & 10 & $9 \%$ & 65 & $59 \%$ & 35 & $32 \%$ \\
\hline 18 & Me siento enfadado/a & 12 & $11 \%$ & 80 & $73 \%$ & 18 & $16 \%$ \\
\hline 19 & Me meto con los demás & 2 & $2 \%$ & 22 & $20 \%$ & 86 & $78 \%$ \\
\hline 20 & Tengo dolor de estómago & 12 & $11 \%$ & 66 & $60 \%$ & 32 & $29 \%$ \\
\hline 21 & Contesto mal a los profesores/as & 2 & $2 \%$ & 12 & $11 \%$ & 96 & $87 \%$ \\
\hline 22 & Falto al instituto porque estoy enfermo/ & & $5 \%$ & 44 & $40 \%$ & 61 & $55 \%$ \\
\hline
\end{tabular}

Fuente: Estudio de estrés académico 
Educación en aulas virtuales

\section{Figura 2}

Preguntas relacionadas con el cuestionario sobre aulas virtuales
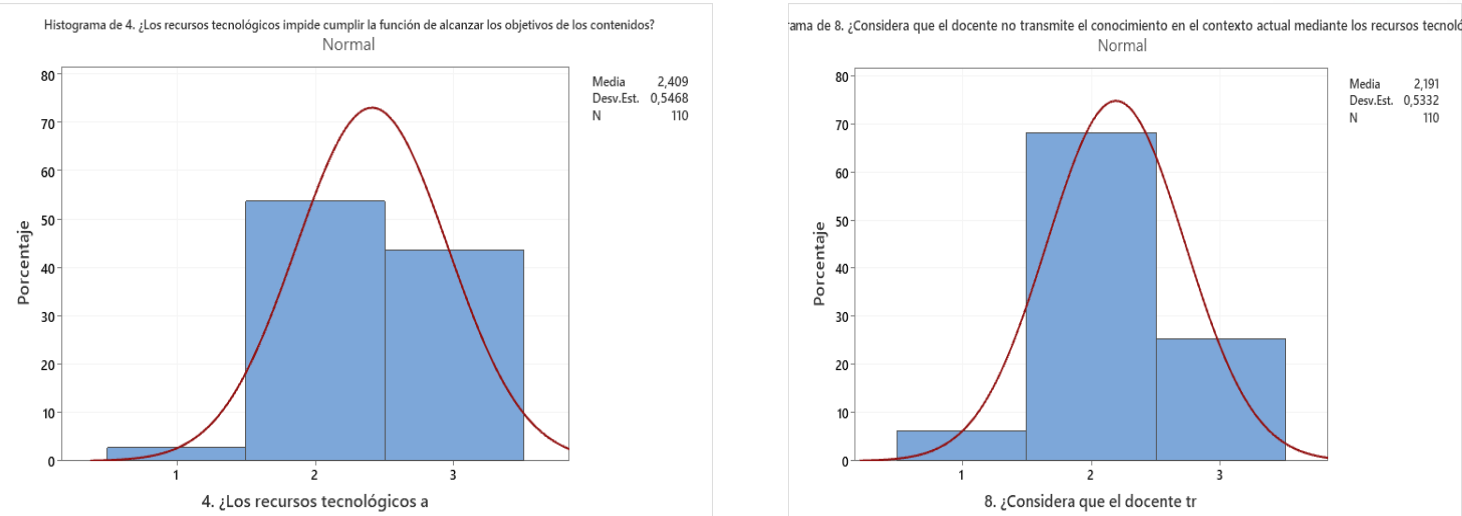

Nota: Altos niveles de estrés académico presentado por el contexto y la falta de conocimiento dentro de los recursos tecnológicos

Fuente: Estudio de educación en las aulas virtuales

La figura 1, muestra que el $55 \%$ corresponde a 60 estudiantes, no se encuentran conformes con respecto a la educación en aulas virtuales, y el $45 \%$ correspondiente a 42 estudiantes muestran estar de acuerdo con la educación virtual impartida en la Universidad en la carrera de Psicopedagogía.

La figura 1, muestra que el $69 \%$ corresponde a 75 estudiantes, no se encuentran conformes con respecto a la educación en aulas virtuales, y el 25\% correspondiente a 27 estudiantes muestran estar de acuerdo con la educación virtual impartida en la Universidad en la carrera de Psicopedagogía.

\section{Tabla 2}

Educación en aulas virtuales

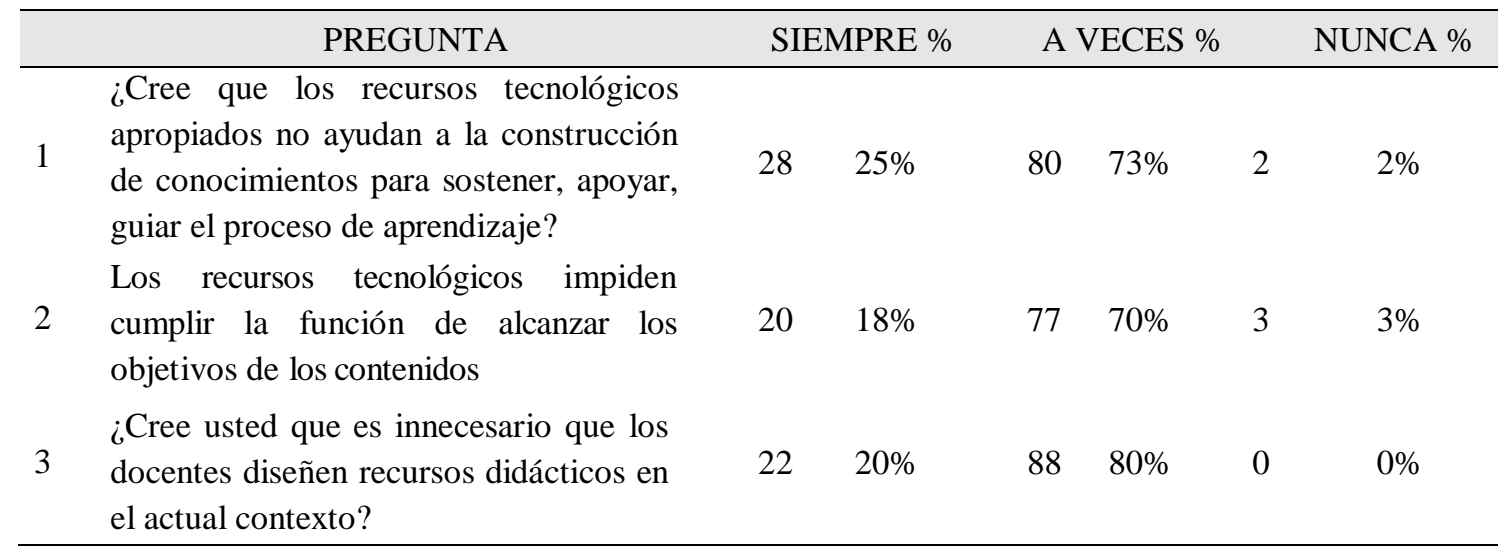




\section{Tabla 2}

Educación en aulas virtuales (continuación)

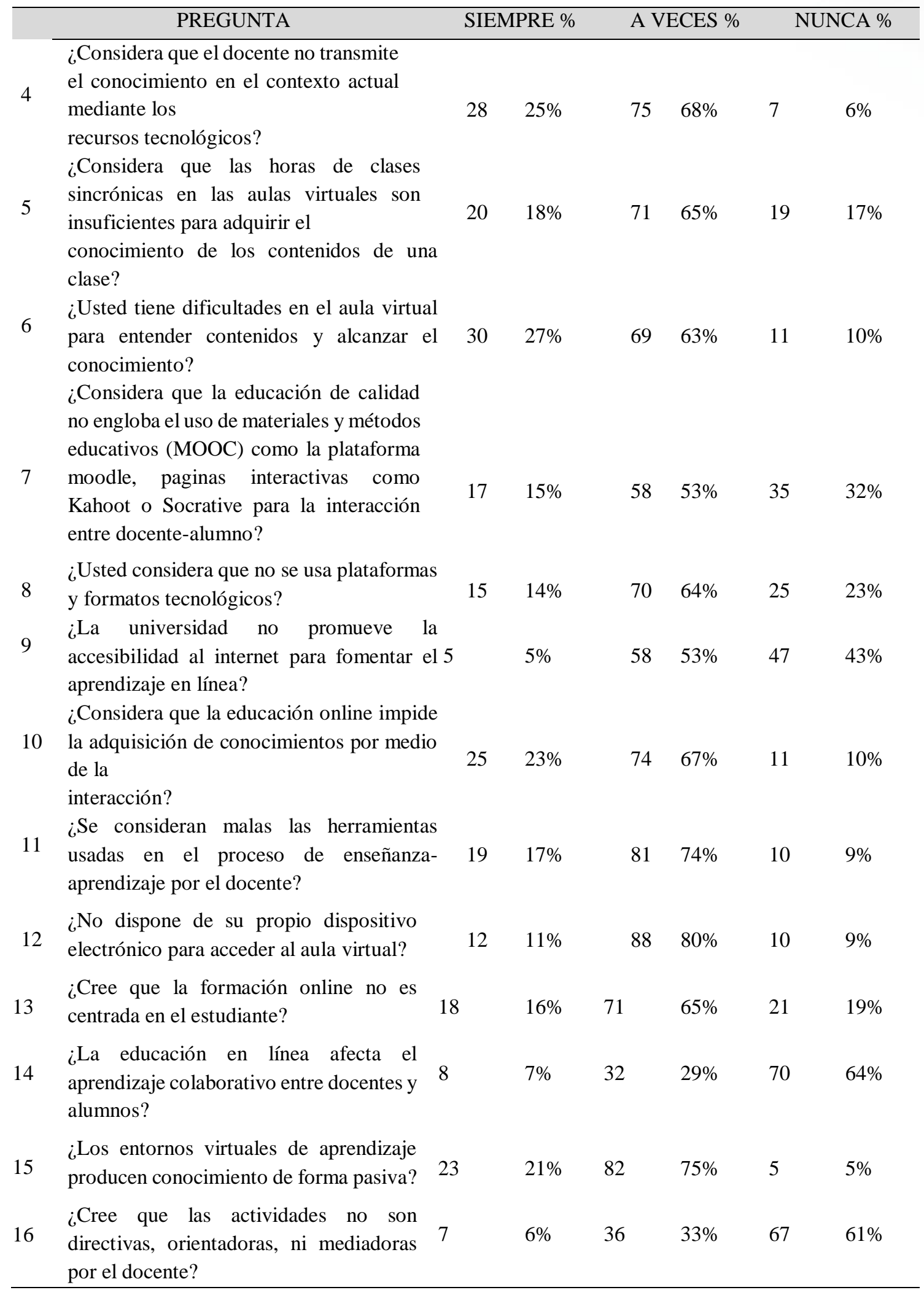




\section{Tabla 2}

Educación en aulas virtuales (continuación)

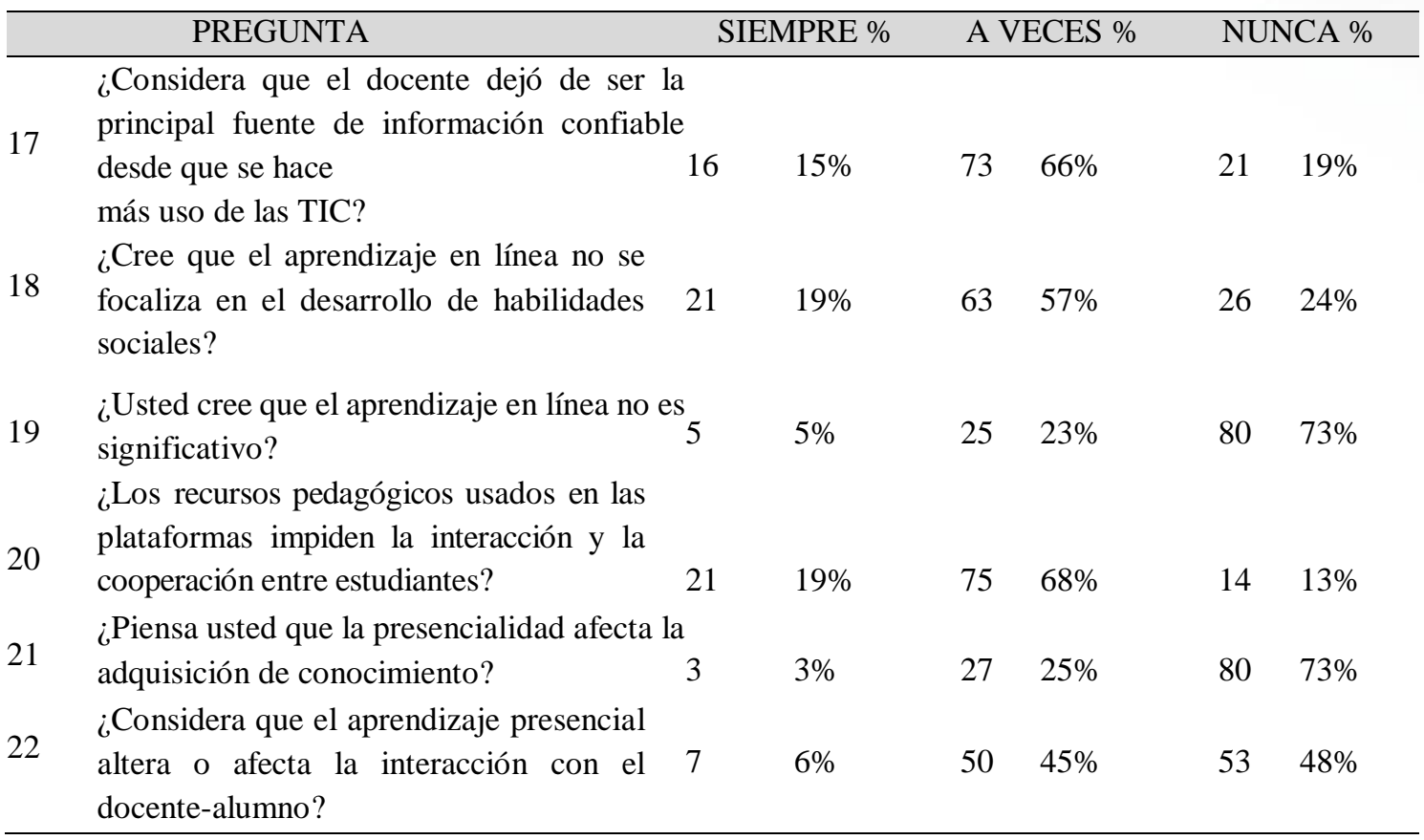

Fuente: Estudio de educación en aulas virtuales (2021)

\section{Figura 3}

Gráfica de correlaciones entre las variables estrés académico y educación en aulas virtuales de Spearman

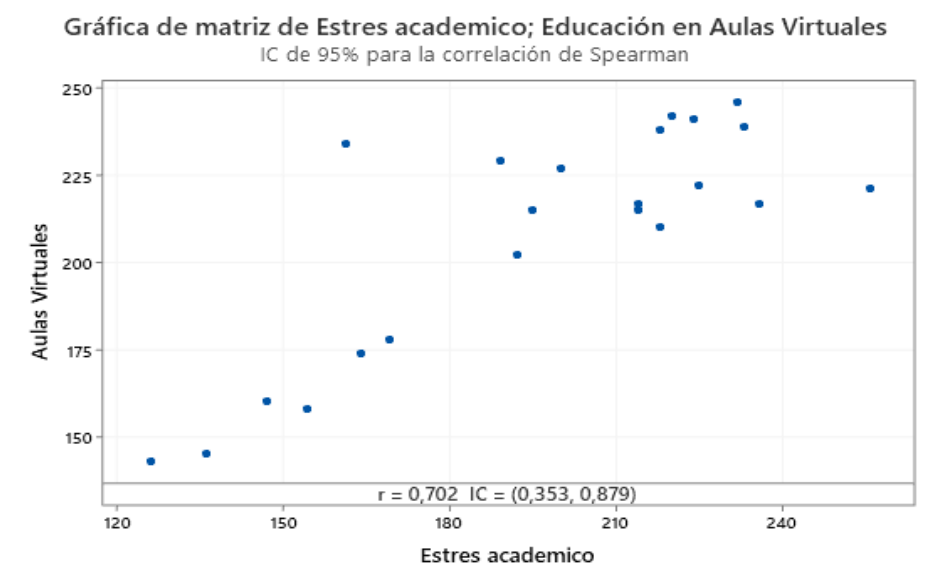

Nota: Nivel de correlación entre el estrés académico y la educación dentro de las aulas virtuales

Fuente: Estudio de estrés académico y la educación en aulas virtuales

La correlación entre el estrés académico y la educación en aulas virtuales es de 0,702; lo cual refleja que los estudiantes de 7 mo semestre de la carrera de psicopedagogía de la 
Universidad Técnica de Ambato, tienen estrés debido a la modalidad virtual en la que desarrollan sus clases, ya que se ha implementado nuevas herramientas tecnológicas, como el uso de plataformas virtuales, actividades educativas multimedia y páginas interactivas. El uso adecuado de estas herramientas tecnológicas permite disminuir el estrés si hay una inducción previa sobre el manejo de estas.

\section{Conclusiones}

- El estrés académico en los estudiantes es alto debido a la exigencia de la modalidad virtual tanto en el uso adecuado de tiempo como en las estrategias metodológicas aplicadas por los profesores.

- El exceso de tiempo frente a un ordenador implica un nivel alto de estrés por cuanto se pierde el contacto humano y al ser parte de una especie gregaria es una necesidad humana el compartir con otros seres humanos en un ambiente educativo humanizado.

- La improvisación de estrategias didácticas por parte de los profesores en la modalidad virtual ha ocasionado que los estudiantes de séptimo semestre no optimicen su tiempo en plataformas virtuales y la falta de capacitación en el uso de herramientas digitales ha tenido como resultado una excesiva pérdida de tiempo en la ejecución de las tareas asignadas para su trabajo autónomo.

\section{Referencias bibliográficas}

Carrasco, O., Caicedo, J., Savedra, O., \& Ochoa, V. (2021). Fundamentos Pedagógicos para la enseñanza-aprendizaje de la Educación Física en Modalidad Virtual: Un reto actual. Ciencia Digital.

Navarrete, F., Sánchez, V., Altamirano, A., \& Cevallos, V. (2019). Utilización de aulas virtuales para el desarrollo de la inteligencia visual-espacial en estudiantes universitarios. Ciencia Digital.

Indacochea Mendoza, L. R., López Pazmiño, M. N., Toasa Galarza, M. E., \& Díaz Núñez,

K. E. (2021). El estrés académico durante la emergencia sanitaria y sus afectaciones en las funciones básicas cognitivas: memoria, comprensión lenguaje, pensamiento. Explorador Digital, $\quad$ 5(2), 101-119. https://doi.org/10.33262/exploradordigital.v5i2.1664

Pérez Esquivel, V., \& Díaz Martín, Y. (2019). Intervención Psicológica para la Gestión del Estrés en Deportistas Juveniles Federados: Intervención Psicológica para la Gestión del Estrés en Deportistas Juveniles Federados. Ciencia Digital, 3(2.5), 105-119. https://doi.org/10.33262/cienciadigital.v3i2.5.535 
Gil, J., Álvarez, A., \& Millán, J. (2019). Organización de un curso en aulas virtuales: fortalezas y debilidades para el aprendizaje. Redalyc.org.

Salvat, B. (2017). La evolución del e-learning: del aula virtual a la red. Redalyc.org.

Sánchez, L. (2020). Impacto del Aula Virtual en el Proceso de Aprendizaje de los

Svensson, V. (2018). Análisis de portadas de aulas virtuales. Redalyc.org.

Ticona, M., Zela, N., \& Vásquez, L. (2021). Ansiedad y estrés en la educación virtual en el periodo de emergencia sanitaria por el COVID-19. Revista científica de sistemas e informática.

Toledo, A. (2019). La tutoría a través de las aulas virtuales: Una experiencia de formación y autoformación. Revista Científica de Publicación del centro Psicopedagógico y de Investigación en Educación Superior.

Berrío, N., \& Mazo, R. (2018). Estrés Académico. Revista de Piscología Universidad de Antioquia.

Emiro, J., Amador, O., \& Castañeda, T. (2020). Estrés académico en estudiantes universitarios. Revista Psico espacios.

Méndez, G., Guamán, M., Sigüenza, W., \& Espinoza, A. (2018). Estudio descriptivo de los sucesos de la vida estresores en adolescentes. Innova Research Journal.

Silva, M., López, J., \& Columba, M. (2020). Estrés académico en estudiantes universitarios. Redalyc.org.

Martínez, G. A., \& Jiménez, N. (2020). Análisis del uso de las aulas virtuales en la Universidad de Cundinamarca, Colombia. Formación universitaria, 13(4), 81-92.

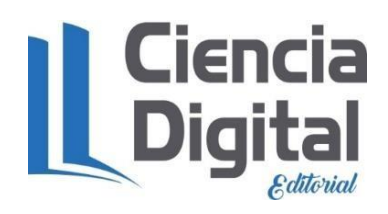


El artículo que se publica es de exclusiva responsabilidad de los autores y no necesariamente reflejan el pensamiento de la Revista Conciencia Digital.

\section{Ciencia \\ LDigital}

El artículo queda en propiedad de la revista y, por tanto, su publicación parcial y/o total en otro medio tiene que ser autorizado por el director de la Revista Conciencia Digital.
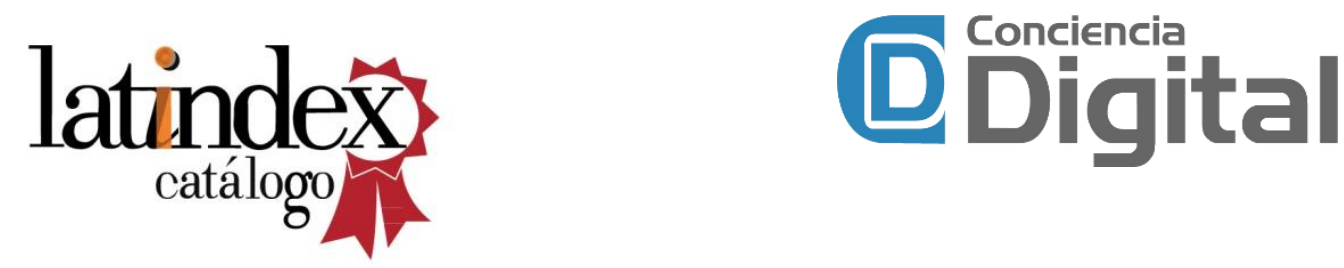

Indexaciones

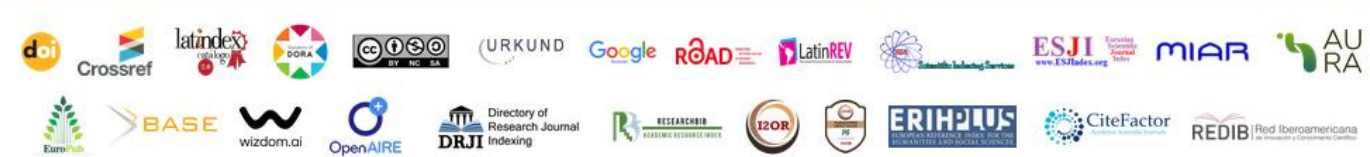

